\title{
Priming atencional en patrones visuales jerárquicos: Efectos del nivel atendido, tamaño absoluto y congruencia estimular
}

\author{
Antonio Prieto $^{1 *}$ y Pedro R. Montoro 2 \\ 1 Departamento de Psicología Básica II, UNED \\ 2 Departamento de Psicología Básica I, UNED
}

\begin{abstract}
Resumen: Estudios previos sobre el procesamiento visual de patrones jerárquicos, muestran que la respuesta ante objetivos presentados en cualquiera de sus niveles (global/local) es más rápida cuando en el ensayo anterior el objetivo se presentó en ese mismo nivel (efecto de priming secuencial). En el presente trabajo, se utiliza un nuevo paradigma de priming atencional para explorar la existencia de este efecto secuencial dentro de cada ensayo en lugar de en diferentes ensayos. Para ello se realizaron dos experimentos en los que se manipuló el tipo de atención (selectiva vs. dividida), la congruencia de los estímulos y el tamaño absoluto de los mismos. Los resultados indican que el efecto de priming secuencial se debe al nivel jerárquico atendido, con independencia del tamaño absoluto de los estímulos, en tareas de atención selectiva (Experimento 1), y que es modulado por el tamaño absoluto de los estímulos tras el cambio a una tarea de atención dividida (Experimento 2). Por último, el efecto de priming secuencial tan solo aparece cuando los estímulos presentados son incongruentes.

Palabras clave: Priming; atención selectiva; atención dividida; estímulos jerárquicos; procesamiento global/local.
\end{abstract}

Title: Attentional priming in hierarchical visual patterns: Effects of treated level, absolute size and consistency stimulate.

Abstract: Previous studies on the visual processing of hierarchical stimuli showed that responses to targets presented either in the local or in the global level were faster when the target was presented at the same hierarchical level as the previous trial (sequential priming effect). In the present work, a new attentional priming paradigm was developed in order to explore this sequential effect by means of a within-trial design. For this purpose, two experiments were conducted manipulating attention (selective vs divided), congruency and stimulus size. Results suggested that the main factor underlying the sequential priming effect was the hierarchical level attended, at least in a selective attention task (Experiment 1). In addition, the stimulus size modulated the sequential priming effect in a divided attention task (Experiment 2). Interestingly, the sequential priming effect was only reliable when incongruent stimuli were presented.

Key words: Priming; selective attention; divided attention; hierarchical stimuli; global/local processing.

\section{Introducción}

La organización de la información visual desempeña un papel fundamental en la percepción. Las propiedades emergentes que surgen de la interacción entre los distintos componentes individuales de figuras complejas, tienen un poderoso efecto en el procesamiento visual (Navon, 1977; Kimchi, 1992; Kinchla y Wolfe, 1979; Palmer, 1980, 1999; Pomerantz, Sager y Stoever, 1977). Un persuasivo ejemplo de este efecto lo encontramos en el procesamiento de patrones jerárquicos formados mediante procesos de agrupamiento perceptivo. Navon (1977), utilizando patrones en los que un conjunto de letras formaba una letra de mayor tamaño, sugirió que la forma global de estos patrones es percibida antes que sus partes componentes. El desarrollo posterior de esta hipótesis de precedencia global propone que el procesamiento perceptivo procede desde el análisis de la forma global hasta sus detalles. De manera complementaria el procesamiento global no solo precede en el tiempo al de los elementos locales, también interfiere sobre estos de manera unidireccional. En otras palabras, mientras que los elementos globales interfieren el procesamiento de los elementos locales lo contrario no parece ocurrir o, al menos, no con la misma magnitud o intensidad (Luna, Merino y Marcos Ruiz, 1990; Pomerantz, 1983; Robertson, Lamb y Zaidel, 1993).

En las décadas posteriores al estudio pionero de Navon, un buen número de trabajos han explorado los determinantes atencionales implicados en el procesamiento de los com-

* Dirección para correspondencia [Correspondence address]: Antonio Prieto. Departamento de Psicología Básica II, Facultad de Psicología (UNED). C/ Juan del Rosal, 10, Ciudad universitaria, 28040, Madrid (España). E-mail: antonioprieto@psi.uned.es ponentes globales y locales en patrones jerárquicos (Arnau, Blanca, Rosel y Salvador, 1992; Flevaris, Bentin y Robertson, 2010; Flevaris Bentin y Robertson, 2011; Lamb y Yund, 1996; Mena, López Montiel y López Montiel, 2006; Robertson 1996, 1999; Robertson, Egly, Lamb y Kerth, 1993; Ward, 1982).

Ward (1982) fue el primero en mostrar que el tiempo de reacción (TR) para detectar un componente de un nivel determinado en un patrón jerárquico, es modulado por el nivel en que el estímulo objetivo había sido presentado en el ensayo anterior. En trabajos posteriores, Robertson y cols. (1993), Lamb y Yund (1996a) y Robertson (1996) profundizaron en el estudio del efecto de priming secuencial descrito por Ward (1982). Sin embargo, ninguno de estos trabajos examinó en qué medida los efectos secuenciales están ligados a atributos físicos absolutos o relativos de la escena visual. Este aspecto fue abordado por Kim, Ivry y Robertson (1999) y Flevaris et al. (2011). En el primero de los trabajos Kim et al. (1999) exploraron la relación entre el priming secuencial, el nivel atendido y el tamaño total del estímulo o resolución. Sus resultados muestran que el efecto de priming secuencial parece estar mediado por el nivel en el que el estímulo aparece, independientemente del tamaño físico absoluto del estímulo excepto bajo circunstancias en las que el tamaño absoluto se mantiene constante (debido a la adición de un marco, o a la inclusión de un nuevo nivel jerárquico que actúa de relleno). Parece, en definitiva, que ambos factores contribuyen a los efectos de priming secuencial, aunque lo hacen bajo circunstancias diferentes. En el segundo de los trabajos mencionados, Flevaris et al. (2011) ampliaron los resultados anteriores a la detección de la orientación de enrejados sinusoidales de diferentes frecuencias espaciales (Campbell y Robson, 1968; Henning, Gevene y Broadbent, 
1975; Lamb, Yund y Pond 1999). Sus resultados sugieren la implicación de un mecanismo de selección del rango relativo de frecuencias espaciales relevante para la tarea independiente del tamaño absoluto de los estímulos.

El presente trabajo pretende estudiar la relación entre el priming secuencial y el procesamiento de estímulos jerárquicos, centrándose en la disociación entre nivel jerárquico atendido y tamaño absoluto de la región atendida del espacio, junto con la influencia de otros factores como la congruencia de los estímulos y el tipo de tarea (atención selectiva vs dividida) en la aparición del efecto de priming secuencial dentro de cada ensayo, en contraste con trabajos anteriores que exploran este efecto entre ensayos (Robertson, 1996; Ward, 1982). Para este fin, se diseñaron dos experimentos, en los que el estímulo utilizado como prime debía ser atendido en uno de sus dos niveles global/local, al mismo tiempo que el estímulo variaba de manera independiente su tamaño absoluto. Si el factor esencial implicado en el efecto de priming secuencial fuese el tamaño absoluto de la región del espacio atendida durante la tarea de priming, las respuestas a los estímulos locales en la segunda de las tareas deberían ser facilitadas en mayor medida por el tamaño pequeño del prime (que posee el mismo tamaño que los estímulos locales de los patrones jerárquicos ulteriores) independientemente del nivel jerárquico al que los participantes atiendan durante la primera tarea, es decir, los efectos de priming secuencial quedarían ligados a las variación del tamaño absoluto del estímulo atendido. Alternativamente, si los efectos de priming secuencial son debidos a la selección atencional previa de uno de los niveles jerárquicos, la segunda tarea debería verse facilitada siempre que, dentro del mismo ensayo, el nivel jerárquico atendido durante la tarea de priming coincida con el atendido posteriormente, independientemente del tamaño total de la región atendida del espacio y del tamaño total del estímulo atendido. En otras palabras, la primera hipótesis predice que las respuestas al nivel local se verán facilitadas por la coincidencia en tamaño absoluto entre los estímulos atendidos durante la tarea de priming y los estímulos objetivos subsiguientes, independientemente del nivel atendido. La segunda hipótesis, por el contrario, predice que las respuestas se verán facilitadas por la coincidencia entre los niveles jerárquicos (global/local) en que los estímulos atendidos aparecen, independientemente de la coincidencia en el tamaño absoluto de los mismos. En el Experimento 1 se utiliza un paradigma de atención selectiva en el que los participantes conocen de antemano la localización (en cuanto a su nivel jerárquico) del estímulo objetivo, mientras que el Experimento 2 introduce un paradigma de atención dividida que impide a los participantes conocer de antemano la localización del estímulo objetivo y el empleo de estrategias de respuesta que puedan debilitar el efecto de priming secuencial, junto con algunas mejoras de diseño derivadas de los resultados obtenidos en el primer Experimento.

\section{Experimento 1}

El principal objetivo del experimento es evaluar la influencia del tamaño absoluto del prime y el nivel jerárquico (local/global) primado (tamaño relativo del prime), sobre la respuesta posterior a objetivos en patrones jerárquicos que pueden coincidir (en relación al prime) tanto en el nivel jerárquico en el que aparecen, como en el tamaño absoluto de los mismos. Para ello se utiliza una tarea de atención selectiva en la que los participantes conocen de antemano el nivel jerárquico en el que aparecerá el estímulo objetivo, y se varía de manera ortogonal tanto el nivel jerárquico primado como el tamaño absoluto del prime. La influencia de la congruencia de los estímulos es también evaluada mediante la inclusión de estímulos congruentes e incongruentes. Especialmente interesante resulta para los objetivos del Experimento la condición en la que el tamaño absoluto total del estímulo utilizado como prime coincide con el tamaño de los elementos locales de los estímulos objetivo posteriores. En esta condición quedan disociados tamaño absoluto y nivel jerárquico atendido, lo que permite aislar el factor o factores involucrados en el efecto de priming secuencial.

\section{Método}

\section{Participantes}

Participaron un total de treinta y seis sujetos de entre 19 y 57 años (edad media: 29.75; desviación típica: 9.52), 32 mujeres y 4 hombres, estudiantes de $2^{\circ}$ curso del Grado en Psicología. Todos los participantes tenían visión normal o corregida. Tres de los participantes eran zurdos, el resto eran diestros.

\section{Estímulos y aparatos}

La secuencia de estímulos fue presentada en monitores de 17 pulgadas con relación de aspecto 5:4 y tasa de refresco de $60 \mathrm{~Hz}$, a una distancia aproximada de $60 \mathrm{~cm}$ y con una resolución de 800 x 600 píxeles. Los monitores estaban controlados por ordenadores personales y toda la presentación fue diseñada y administrada mediante el paquete informático E-Prime 1.2 (Psychology Software Tools, 1996-2002).

Para la realización de la tarea go/no-go utilizada como prime se emplearon los estímulos que aparecen en el panel A de la Figura 1. Estos consistieron en nueve patrones jerárquicos de tres tamaños distintos ( $97 \times 97$ píxeles, $3.82^{\circ}$ a.v.; $48 \times 48$ pix, $2.10^{\circ}$ a.v. y $12 \times 12$ pix, $0.48^{\circ}$ a.v.). El área ocupada por los estímulos locales en el tamaño grande igualó en tamaño al área total de los estímulos de menor tamaño (12 x 12 píxeles), y los estímulos de tamaño intermedio ocuparon aproximadamente la cuarta parte del área ocupada por los de mayor tamaño. Para la realización de esta primera tarea se construyeron tres puntos de fijación, consistentes en cruces con tamaños iguales a los posibles tamaños de los estímulos presentados. En la tarea de identificación global/local, los estí- 
mulos presentados fueron los que se muestran en el panel B de la Figura 1. Todos los estímulos fueron diseñados mediante el programa Microsoft Paint y se presentaron en color negro sobre fondo "silver", según la paleta de colores del paquete informático E-Prime (gris 25\%, RGB: 195).

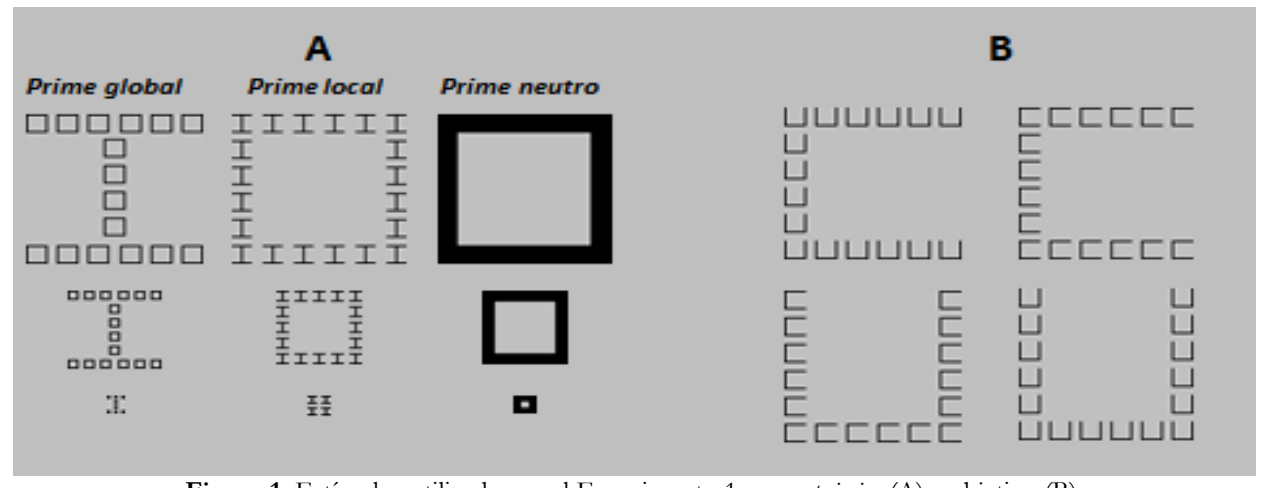

Figura 1. Estímulos utilizados en el Experimento 1 como priming (A) y objetivo (B).

\section{Diseño y procedimiento}

Se utilizó un diseño factorial 3 × 2 × 2 × 2 que incluyó cuatro factores intrasujeto: tamaño absoluto del prime con tres niveles (grande, mediano y pequeño), nivel jerárquico primado con dos niveles (global, local), nivel jerárquico en el que apareció el estímulo objetivo con dos niveles (global, local) y congruencia del estímulo con dos niveles (congruente e incongruente). En el caso del último factor, los estímulos congruentes fueron aquellos formados por las mismas letras en los niveles local y global. Los incongruentes fueron los formados por letras distintas en los niveles local y global.

El experimento fue dividido en dos partes, cada una de ellas correspondiente a un nivel del factor nivel jerárquico en el que apareció el estímulo objetivo (global, local). En cada parte se instruyó a los sujetos para que respondieran pulsando la tecla correspondiente a la letra aparecida en el nivel objetivo (teclas 1 y 2 del teclado numérico) ignorando el otro nivel. La tarea go/no go que se utilizó como prime se realizó de manera idéntica en ambos bloques, y en ella se instruyó a los sujetos para que pulsaran la barra espaciadora siempre que en pantalla apareciese una letra " $\mathrm{I}$ " independientemente del nivel jerárquico en el que esta apareciese. Cada parte estuvo compuesta por una fase de práctica formada por 36 ensayos, seguida de 4 bloques experimentales de 72 ensayos cada uno. El número total de ensayos fue de $648 \mathrm{y}$ el orden de presentación de las dos partes fue contrabalanceado. Los sujetos realizaron la tarea en una única sesión de manera individual y recibieron indicaciones para responder rápidamente intentando cometer la menor cantidad de errores posible.

En cada ensayo, tras la desaparición del punto de fijación, se presentaba uno de los estímulos para la realización de la tarea go/no go durante $150 \mathrm{~ms}$, seguido de una pantalla en blanco de $1000 \mathrm{~ms}$ de duración durante la cual los sujetos debían responder pulsando la barra espaciadora con el dedo pulgar de su mano dominante en el caso de que el estímulo presentado contuviese una letra "I" (respuesta go); en caso contrario no debían emitir respuesta alguna (respuesta no-go). A continuación se presentaban el estímulo objetivo durante $150 \mathrm{~ms}$, seguido de una pantalla en blanco que permanecía hasta que el sujeto consignase su respuesta mediante las teclas " 1 " y "2" del teclado numérico del ordenador (contrabalanceadas para cada participante). La secuencia experimental completa se muestra en la Figura 2.

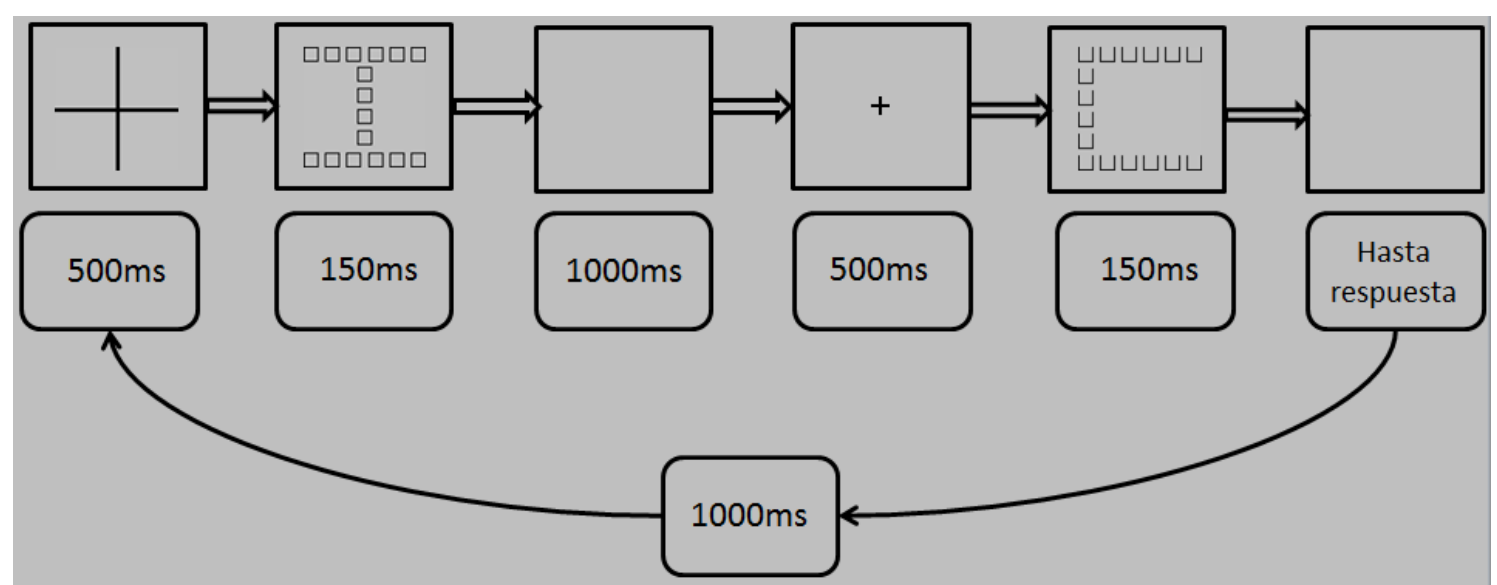

Figura 2. Secuencia experimental del Experimento 1. 


\section{Resultados}

El porcentaje promedio de respuestas correctas para la tarea go/ no go utilizada como prime fue de un 98\% (DT = 1.41), mientras que para la tarea de identificación con patrones jerárquicos tipo Navon este porcentaje fue del $96 \%$ (DT $=2)$. Dos de los 36 participantes en el experimento no alcanzaron el $90 \%$ de aciertos en alguna de las tareas y fueron excluidos del análisis.

Para el análisis de las respuestas al estímulo objetivo solo se tuvieron en cuenta las respuestas "go", puesto que son aquellas en las que el participante tuvo que dirigir su atención a un nivel jerárquico concreto.
Los TRs para la tarea de identificación con patrones jerárquicos se analizaron mediante un $\operatorname{ANOVA}(3 \times 2 \times 2 \times 2)$ de medidas repetidas. Se eliminaron los TRs por debajo de $200 \mathrm{~ms}$ para asegurar que ninguna respuesta analizada pudiese haberse iniciado antes de la aparición de estímulo objetivo en pantalla. Los TRs por encima de $1500 \mathrm{~ms}$ también fueron eliminados del análisis para evitar la implicación de procesos cognitivos diferentes de los puramente atencionales y perceptivos, así como para evitar la dilución del efecto de primacía global/local (Marí-Beffa, Fuentes, Catena \& Houghton, 2000; Mayas, Fuentes y Ballesteros, 2012; Montoro y Luna, 2009). El número de ensayos eliminados supuso el $0.73 \%$ del total (véase Tabla 1).

Tabla 1. Medias y desviaciones típicas en ms Experimento 1 . TP = tamaño del prime; $\mathrm{NP}=$ nivel primado; $\mathrm{NO}=$ nivel de aparición del objetivo; $\mathrm{CO}=$ congruencia.

\begin{tabular}{|c|c|c|c|c|c|c|c|c|c|}
\hline \multirow[b]{3}{*}{ TP } & \multirow{3}{*}{$\begin{array}{l}\mathrm{NO} \\
\mathrm{CO} \\
\mathrm{NP}\end{array}$} & \multicolumn{4}{|c|}{ Global } & \multicolumn{4}{|c|}{ Local } \\
\hline & & \multicolumn{2}{|c|}{ Congruente } & \multicolumn{2}{|c|}{ Incongruente } & \multicolumn{2}{|c|}{ Congruente } & \multicolumn{2}{|c|}{ Incongruente } \\
\hline & & Media & Dsv.tip & Media & Dsv.tip & Media & Dsv.tip & Media & Dsv.tip \\
\hline \multirow[t]{2}{*}{ Grande } & Global & 507 & 110 & 531 & 112 & 505 & 81 & 546 & 90 \\
\hline & Local & 502 & 98 & 544 & 109 & 502 & 92 & 539 & 81 \\
\hline \multirow[t]{2}{*}{ Mediano } & Global & 505 & 92 & 535 & 105 & 494 & 80 & 539 & 83 \\
\hline & Local & 500 & 97 & 541 & 111 & 497 & 86 & 530 & 75 \\
\hline \multirow[t]{2}{*}{ Pequeño } & Global & 498 & 100 & 524 & 105 & 509 & 90 & 539 & 86 \\
\hline & Local & 503 & 96 & 537 & 102 & 506 & 94 & 527 & 82 \\
\hline
\end{tabular}

El análisis de los TRs reveló un efecto principal estadísticamente significativo para el factor congruencia, $F(1,33)=$ 132.474, $p<.001 ; \eta_{p}^{2}=.801$ (TR congruentes: $503 \mathrm{~ms}$; TR incongruentes: $538 \mathrm{~ms}$ ). Ningún otro efecto principal mostró diferencias estadísticamente significativas.

La interacción de segundo orden entre el nivel primado, nivel de aparición del objetivo y congruencia resultó significativa, $F$ $(1,33)=6.310, p=.017, \eta_{p}^{2}=.161$. Para su análisis se realizaron dos ANOVAs $3 \times 2 \times 2$ de medidas repetidas, tomando por separado estímulos congruentes e incongruentes. Este análisis matiza la interacción significativa de primer orden encontrada entre el nivel primado y el nivel jerárquico de aparición

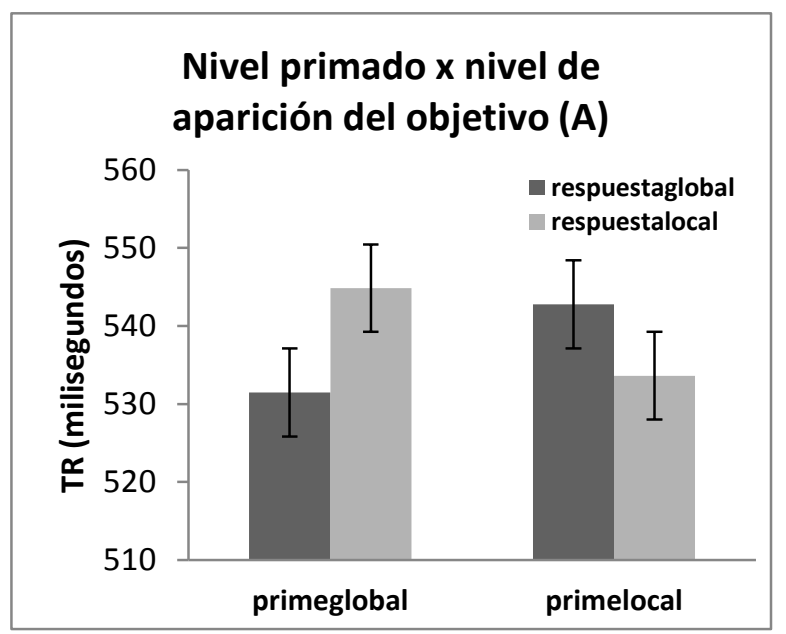

del estímulo objetivo $F(1,33)=9.788 ; p=.004, \eta_{p}^{2}=.229$. Así, cuando el nivel de aparición del objetivo fue el global, las respuestas de los sujetos fueron significativamente más rápidas cuando fueron precedidas de un estímulo que primase el nivel global $(532 \mathrm{~ms})$ respecto a las mismas respuestas precedidas de priming del nivel local $(543 \mathrm{~ms})$, pero solo para los estímulos incongruentes $F(1,33)=4.554, p=.040, \eta_{p}{ }^{2}=$ .121 (Figura 3). Lo mismo ocurrió para los objetivos a nivel local si previamente se primó el nivel local $(534 \mathrm{~ms})$ respecto a las respuestas cuando se primó el nivel global (545 ms). F $(1,33)=.736, p=.014, \eta_{p}^{2}=.170$ (Figura 3).

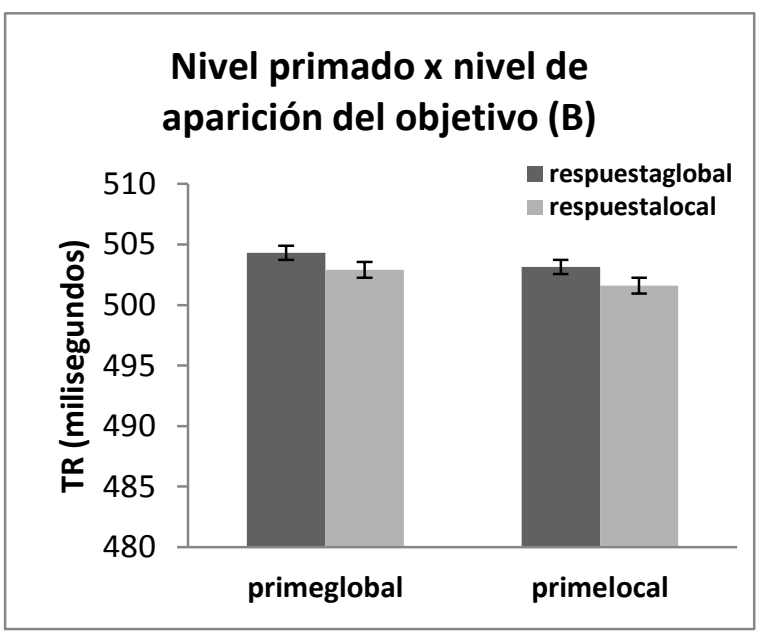

Figura 3. Tiempos de reacción del Experimento 1 en ms, (A) incongruentes, (B) congruentes. 
Debido al especial interés de la condición experimental con tamaño pequeño del prime, en el que el tamaño el estímulo global posee iguales dimensiones que los estímulos locales del patrón jerárquico, se realizó un análisis separado para este nivel del factor mediante un ANOVA 2 × 2 de medidas repetidas manteniendo tan solo los estímulos incongruentes. Los factores fueron nivel primado (global/local) y nivel jerárquico en el que apareció el estímulo objetivo (global/local). Los resultados replican en esencia los hallados en el análisis general, encontrándose un efecto interactivo entre el nivel primado y el nivel jerárquico en el que apareció el estímulo objetivo $F$ $(1,33)=11.962, p=.002, \eta_{p}^{2}=.266$ (Figura 4), indicativo de

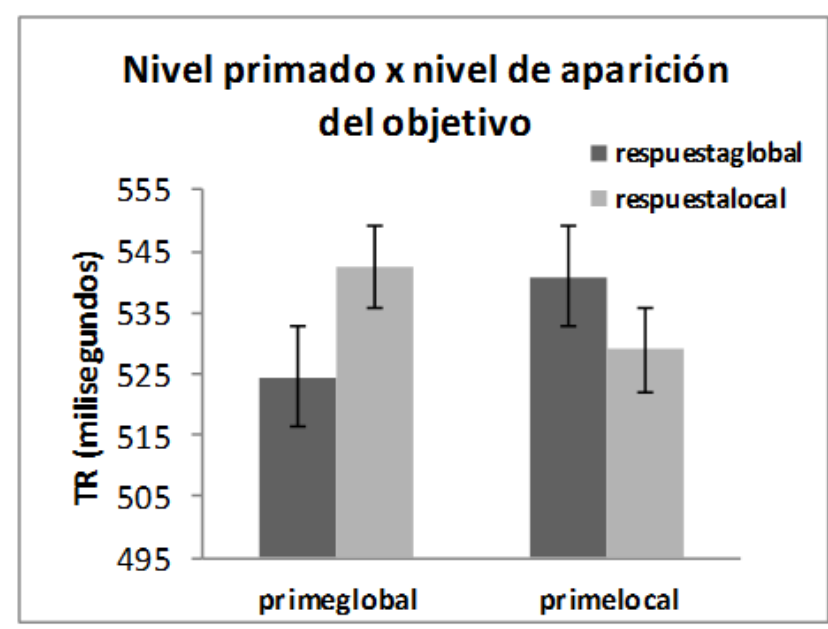

Figura 4. Tiempos de reacción del Experimento 1 en ms. para el tamaño pequeño del prime. Solo estímulos incongruentes. una mayor rapidez de respuesta ante objetivos en el nivel local $(529 \mathrm{~ms})$ cuando ese prima este mismo nivel, respecto a las mismas respuestas precedidas de priming global (542 ms). El mismo patrón para las respuestas globales resultó marginalmente significativo $(p=.073)$.

Un resultado inesperado fue la ausencia del efecto de primacía global habitualmente encontrado en este tipo de tareas, resultado que podría enmascarar diferencias significativas entre las diferentes condiciones experimentales. Una causa hipotética de esta ausencia podría ser la tendencia a la inversión de los TRs asociados a las respuestas globales y locales en función del nivel primado con anterioridad. Para explorar este efecto se construyó una variable diferencial a partir de la sustracción de los TRs asociados a los objetivos globales de los TRs asociados a los objetivos locales (Tabla 2).

Esta nueva variable $\left(\mathrm{TR}_{\text {local }}-\mathrm{TR}_{\text {global }}\right)$, refleja la ventaja en $\mathrm{ms}$ de las respuestas a objetivos globales respecto a las respuestas a objetivos locales. Una vez calculada, se realizó un nuevo ANOVA $3 \times 2 \times 2$ de medidas repetidas con los factores tamaño del prime, nivel primado y congruencia. Los resultados de ANOVA revelan una interacción significativa entre el $n i$ vel primado y la congruencia de los estímulos jerárquicos $F(1,33)$ $=4.906, p=.034, \eta_{p}^{2}=.129$ que acota el efecto principal encontrado del factor nivel primado $F(1,33)=6.634, p=.015$, $\eta_{\mathrm{p}}{ }^{2}=.167$. En este caso, la ventaja en la respuesta a los objetivos globales encontrada cuando el nivel primado es el glo$\mathrm{bal}(+12 \mathrm{~ms})$ se invierte para pasar a ser ventaja local (- 9 $\mathrm{ms}$ ), pero solo para los estímulos incongruentes (Figura 5).

Tabla 2. Medias y desviaciones típicas en ms para la variable Trlocal-Trglobal en el Experimento 1 . TP $=$ tamaño del prime; $\mathrm{NP}=$ nivel primado; $\mathrm{CO}=$ congruencia.

\begin{tabular}{|c|c|c|c|c|c|c|c|c|}
\hline \multirow{3}{*}{$\begin{array}{l}\mathrm{NP} \\
\mathrm{CO} \\
\mathrm{TP}\end{array}$} & \multicolumn{4}{|c|}{ Global } & \multicolumn{4}{|c|}{ Local } \\
\hline & \multicolumn{2}{|c|}{ Congruente } & \multicolumn{2}{|c|}{ Incongruente } & \multicolumn{2}{|c|}{ Congruente } & \multicolumn{2}{|c|}{ Incongruente } \\
\hline & Media & Dsv.tip & Media & Dsv.tip & Media & Dsv.tip & Media & Dsv.tip \\
\hline Grande & -2 & 70 & 15 & 72 & 0 & 74 & -5 & 79 \\
\hline Mediano & -11 & 62 & 5 & 73 & -3 & 56 & -12 & 94 \\
\hline Pequeño & 11 & 71 & 15 & 62 & 2 & 76 & -10 & 73 \\
\hline
\end{tabular}
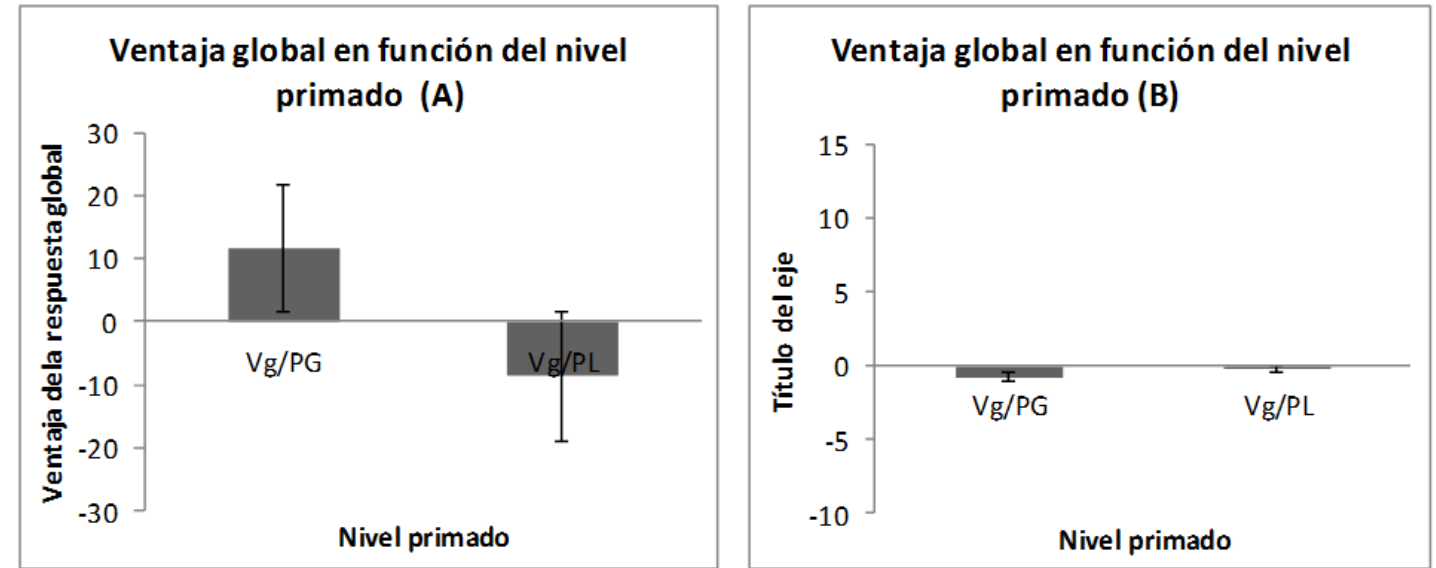

Figura 5. Variable diferencial Experimento 1: $\mathrm{Vg}=$ ventaja global, $\mathrm{PG}=$ priming global, $\mathrm{PL}=$ Priming local, (A) incongruentes, (B) congruentes. 


\section{Discusión}

Los resultados de este primer experimento indican que los efectos facilitadores solo se encuentran cuando existe coincidencia entre los niveles jerárquicos del prime y el estímulo objetivo dentro del mismo ensayo, con independencia del tamaño absoluto de los mismos, en línea con los resultados de los Experimentos 1a y 1b del trabajo de Kim et al. (1999). El efecto, por tanto, no depende de la coincidencia en tamaño absoluto de la región espacial atendida entre el estímulo primante y el estímulo primado (objetivo). Por otra parte, el patrón de efectos descrito solo aparece para los estímulos incongruentes, lo que sugiere que el efecto primante encontrado depende específicamente de la capacidad de la tarea de priming atencional para reducir el grado de procesamiento del nivel jerárquico no atendido, lo que redundaría en una facilitación de la respuesta al objetivo cuando el nivel jerárquico atendido en la tarea de priming coincide con el nivel jerárquico en el que aparece el estímulo objetivo (debido a la menor grado de procesamiento del nivel no atendido) y, alternativamente, en un aumento de la interferencia cuando el nivel jerárquico en el que aparece el objetivo no coincide con el nivel primado anteriormente. Si, por el contrario, el efecto se debiese tan solo a una facilitación del procesamiento del nivel jerárquico primado, se esperaría un efecto similar tanto en estímulos congruentes como incongruentes. En conjunto el patrón de resultados sugiere que ambos niveles jerárquicos son procesados en paralelo por el sistema perceptivo, y que la tarea de priming atencional "inhibe" en cierta forma el procesamiento del nivel jerárquico no atendido.

La realización de un análisis separado (limitado a los estímulos incongruentes) para la condición en la que el tamaño absoluto del prime coincide con el tamaño local de los patrones jerárquicos, coincide en sus resultados con los análisis anteriores. De nuevo la facilitación/interferencia se produce en función del nivel atendido durante la tarea de priming con independencia del tamaño absoluto del prime.

El cálculo de una variable diferencial definida como la ventaja en milisegundos de la respuesta al nivel global, nos permite explorar esta tendencia con mayor precisión. Los resultados del análisis de esta variable replican y amplían los obtenidos anteriormente. La ventaja en términos de TR cuando se responde a un estímulo global precedido de priming de ese mismo nivel, pasa a ser ventaja para las respuestas a estímulos locales cuando el nivel primado con anterioridad es el local. De nuevo este efecto es tan solo significativo para los estímulos incongruentes.

A pesar de que los resultados presentados son congruentes con los encontrados por otros autores (Flevaris et al. 2011; Kim et al. 1999), el diseño del Experimento 1 presenta algunas carencias que impiden una interpretación inequívoca de los resultados. En primer lugar, el hecho de utilizar una tarea de atención selectiva permite a los participantes utilizar diversas estrategias anticipatorias que podrían debilitar o alterar la tarea de priming utilizada. En segundo lugar, los estí- mulos congruentes así como el tamaño intermedio del prime no parecen tener relevancia en los resultados obtenidos. Para resolver los problemas anteriores se diseñó un nuevo experimento en el que se modificó el paradigma atencional de la tarea, con el objetivo de comprobar si tras esta modificación el nivel jerárquico atendido continúa siendo responsable de los efectos de priming secuencial con independencia del tamaño absoluto de los estímulos atendidos.

\section{Experimento 2}

El Experimento 2 pretende replicar los resultados del Experimento anterior haciendo uso de un paradigma de atención dividida, en el que los participantes desconocen en qué nivel aparecerá el estímulo objetivo. Este cambio de tarea persigue que los participantes no puedan anticipar el nivel jerárquico en el que aparecerá el estímulo y eliminar así posibles estrategias atencionales que puedan afectar a los efectos de priming secuencial encontrados. Se añaden además algunas mejoras procedimentales, como una leve variación de la tarea go/no go junto a la eliminación de los estímulos congruentes y el tamaño intermedio del prime.

\section{Método}

\section{Participantes}

Participaron un total de treinta y siete sujetos de entre 19 y 55 años (edad media: 30.38; desviación típica: 10.04), 24 mujeres y 13 hombres, estudiantes de Grado en Psicología. Todos los participantes tenían visión normal o corregida. Uno de los participantes era zurdo, los treinta y seis restantes diestros.

\section{Estímulos y aparatos}

Se utilizaron los mismos aparatos descritos en el Experimento 1.

Para la realización de la tarea go/no go utilizada como prime se emplearon los estímulos que aparecen en la Figura 6A. Estos consistieron en diez patrones jerárquicos de dos tamaños distintos $(97 \times 97$ píxeles, 3.82 a.v. y 12 x 12 píxeles, $0.48^{\circ}$ a.v.). La relación de tamaños y la construcción de los puntos de fijación se mantuvo igual a la utilizada en el Experimento 1, con la excepción del tamaño intermedio del prime que no fue utilizado. En el caso de la tarea de identificación posterior los estímulos consistieron en ocho patrones jerárquicos con un tamaño de 97 x 97 píxeles, formados por letras "C", "U", "D" y "L" en las que siempre apareció una letra "U" o una letra "C" en alguno de los dos niveles (objetivo) combinado con alguna de las otras dos letras (distractor). Véase Figura 6B. El resto del diseño y presentación fue en todo punto igual al utilizado en el Experimento 1. 


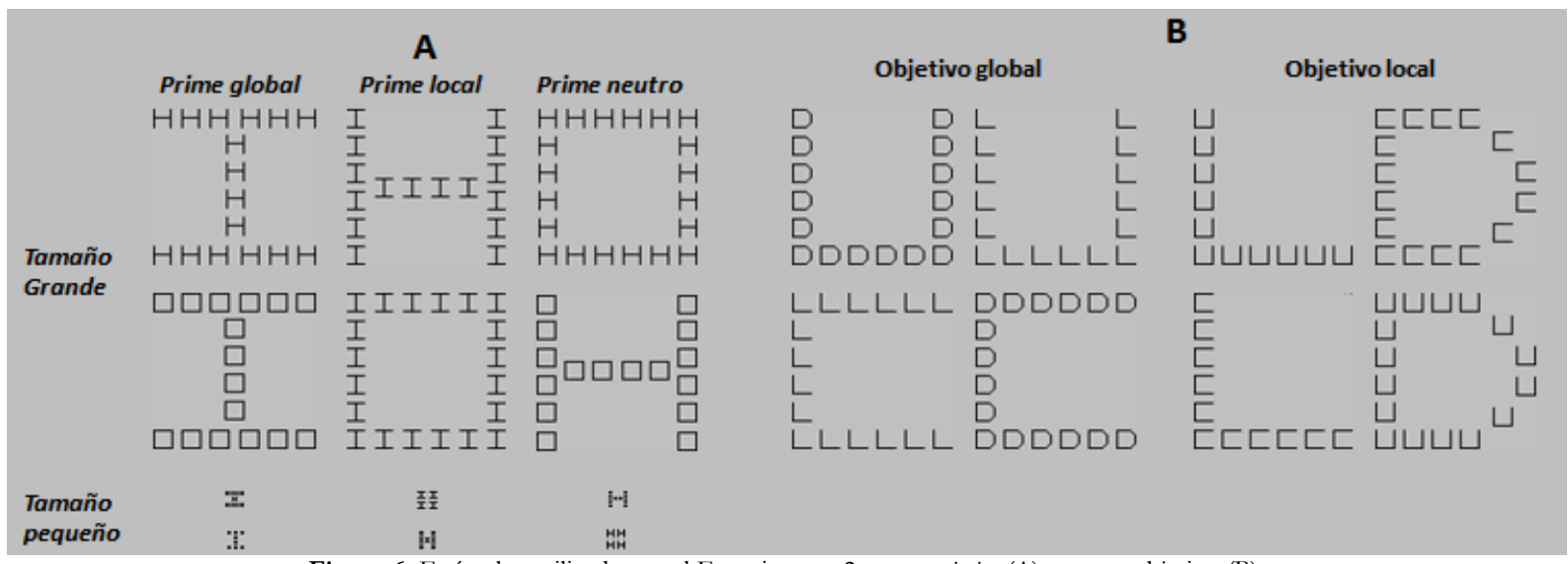

Figura 6. Estímulos utilizados en el Experimento 2 como priming (A) y como objetivo (B).

\section{Diseño y procedimiento}

Se utilizó un diseño factorial $2 \times 2 \times 2$ que incluyó tres factores intrasujeto: tamaño del prime con dos niveles (grande y pequeño), nivel jerárquico primado con dos niveles (global y local) y nivel en el que apareció el estímulo objetivo (global, local). Para la tarea go/no go que se utilizó como prime se instruyó a los sujetos para que identificasen la aparición de una letra "I" independientemente del nivel en el que apareciese. Los participantes tan solo tuvieron que responder ante los patrones jerárquicos posteriores en el caso de que la letra objetivo "I" apareciese durante la primera parte del ensayo. Tras ello los participantes debían identificar la letra objetivo que apareciese en cada ensayo y que, siguiendo un paradigma de atención dividida, podía aparecer tanto en el nivel global como en el nivel local. El experimento consistió en un primer bloque de práctica seguido de la fase experimental que estuvo compuesta de 3 bloques experimentales de 160 ensayos cada uno. Las teclas de respuesta asignadas ("1" y " 2 " del teclado numérico) fueron contrabalanceadas. Las instrucciones a los participantes fueron las mismas que en el Experimento 1.

En cada ensayo, después del punto de fijación se presentó uno de los estímulos para la realización de la tarea go/ no go (150 ms) seguido de una pantalla en blanco de $1000 \mathrm{~ms}$ de duración. Tras un nuevo punto de fijación $(500 \mathrm{~ms})$ se presentaron los estímulos objetivo durante $150 \mathrm{~ms}$, seguidos de una pantalla en blanco de $2000 \mathrm{~ms}$ de duración durante la que el sujeto emitía su respuesta. La secuencia experimental completa puede verse en la Figura 7.

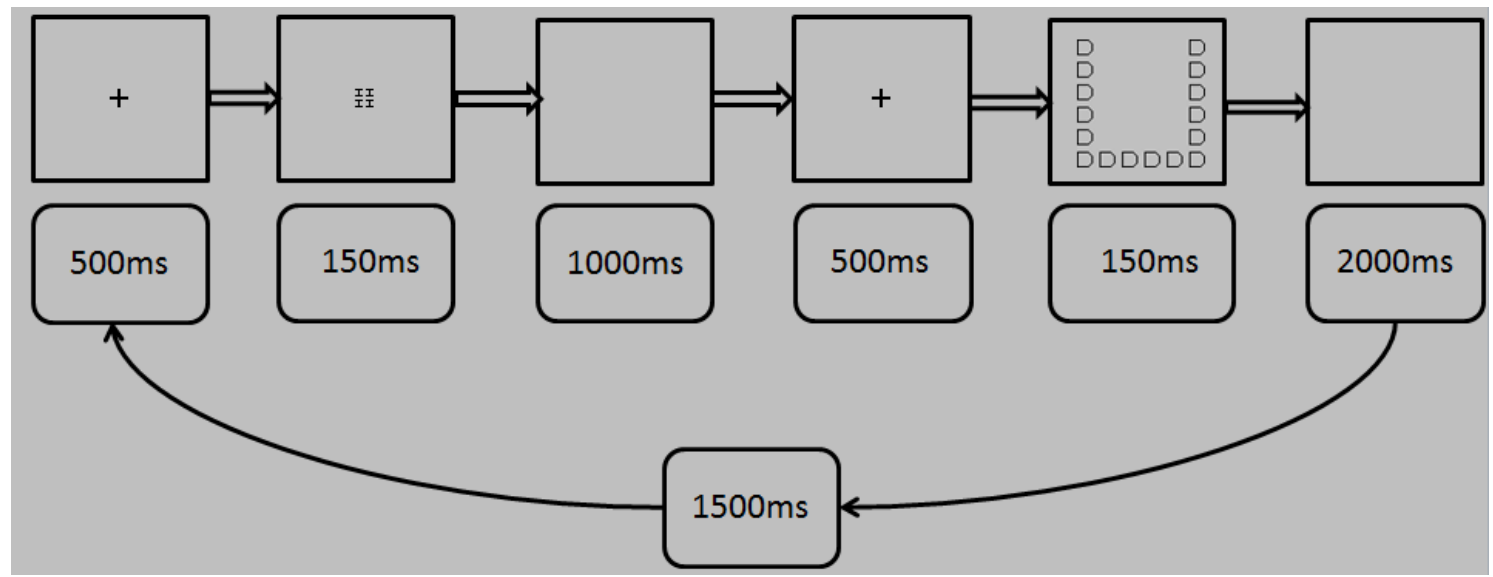

Figura 7. Secuencia experimental del Experimento 2.

\section{Resultados}

El porcentaje promedio de respuestas correctas para la tarea de identificación con patrones jerárquicos alcanzó el $95 \%(D T=2.45)$. Cinco de los 37 participantes en el experimento no alcanzaron el $90 \%$ de aciertos en la tarea y fueron excluidos de los análisis posteriores.
Los TRs para la tarea de identificación con patrones jerárquicos se analizaron mediante un ANOVA $(2 \times 2 \times 2)$ de medidas repetidas. Los TRs para las respuestas incorrectas así como aquellos inferiores a $200 \mathrm{~ms}$ o superiores a 1500 ms fueron eliminados del análisis siguiendo los mismos criterios del Experimento 1. El porcentaje de ensayos eliminados fue del $0.75 \%$ del total, (véase Tabla 3 ). 
Tabla 3. Medias y desviaciones típicas en ms para los TRs en el Experimento 2. $\mathrm{TP}=$ tamaño del prime; $\mathrm{NP}=$ nivel primado; $\mathrm{NO}=$ nivel de aparición del objetivo.

\begin{tabular}{|c|c|c|c|c|c|}
\hline \multirow[b]{2}{*}{$\underline{T P}$} & \multirow{2}{*}{$\frac{N O}{\underline{N P}}$} & \multicolumn{2}{|c|}{ Global } & \multicolumn{2}{|c|}{ Local } \\
\hline & & Media & Dsv.tip & Media & Dsv.tip \\
\hline \multirow[t]{2}{*}{ Grande } & Global & 735 & 129 & 794 & 135 \\
\hline & Local & 753 & 132 & 779 & 124 \\
\hline \multirow[t]{2}{*}{ Реqueño } & Global & 741 & 133 & 771 & 120 \\
\hline & Local & 747 & 141 & 781 & 131 \\
\hline
\end{tabular}

El análisis de los TRs reveló un efecto principal significativo para el factor nivel de aparición del estímulo objetivo $\mathrm{F}(1,31)$ $=12.345, p<.001, \eta_{p}^{2}=0.285$, respondiendo los participantes más rápido a los objetivos que aparecieron en el nivel global (744 ms) respecto a los objetivos que aparecen en el nivel local (782 ms). Más interesante resulta la interacción de segundo orden encontrada entre los factores tamaño del prime, nivel primado y nivel de aparición del estímulo objetivo $F(1,31)=$ 12.546, $p<.001, \eta_{p}^{2}=.288$. Las comparaciones post-hoc realizadas (aplicando ajuste Bonferroni) reflejan que las respuestas ante los objetivos aparecidos en el nivel global son más rápidas si se ha primado previamente ese nivel $(735 \mathrm{~ms})$, respecto a las respuestas en ese mismo nivel cuando fueron precedidas de priming del nivel local $(753 \mathrm{~ms}), F(1,31)=$ $4.520, p=.042, \eta_{p}^{2}=0.127$. Por su parte las respuestas ante estímulos aparecidos en el nivel local fueron más rápidas cuando fueron precedidas de priming del nivel local (778 ms), comparadas con las respuestas al nivel local pero precedidas de priming del nivel global $(794 \mathrm{~ms}), F(1,31)=4.874, p=$ $.035, \eta_{p}{ }^{2}=.136$. En ambos casos esto solo ocurrió cuando el tamaño absoluto del prime era el mayor de los dos posibles (véase Figura 8).
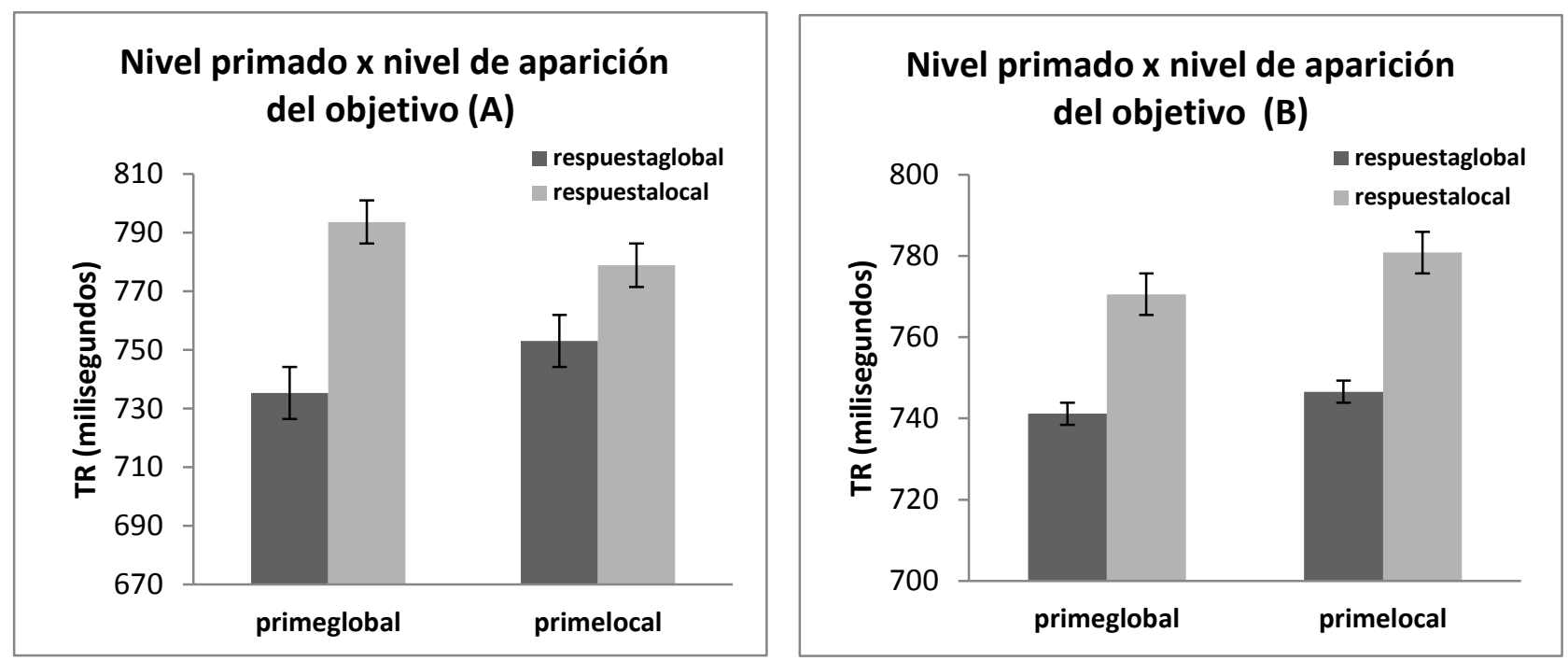

Figura 8. TRs medios del Experimento 2 en ms, para tamaño grande (A) y pequeño (B) del prime.

De nuevo el análisis de los TRs puede enmascarar la modificación de la ventaja global debida a las distintas condiciones de priming. Se calculó una variable diferencial siguiendo un procedimiento idéntico al detallado en el Experimento 1 ( $\left.\mathrm{TR}_{\text {local }}-\mathrm{TR}_{\text {global }}\right)$, junto con un nuevo ANOVA $2 \times 2$ de medidas repetidas con los factores tamaño absoluto del prime y nivel primado. Los resultados muestran un efecto significativo de la interacción entre tamaño del prime y nivel primado $F(1,31)=12.505, p<.001, \eta_{p}^{2}=.287$. Las comparaciones post-hoc realizadas (corrección Bonferroni) muestran una mayor ventaja ( $+58 \mathrm{~ms})$ de los objetivos en el nivel global precedidos de priming de ese mismo nivel limitada al tamaño absoluto mayor del prime, respecto a la ventaja global cuando previamente se ha primado el nivel local ( $+26 \mathrm{~ms})$.

Por otra parte, la ventaja global cuando se ha primado el nivel global es mayor ( $+58 \mathrm{~ms}$ ) cuando el prime es el de mayor tamaño absoluto, respecto a la misma ventaja global cuando el tamaño absoluto del prime es el menor (+ $29 \mathrm{~ms})$ (véase Figura 9). 


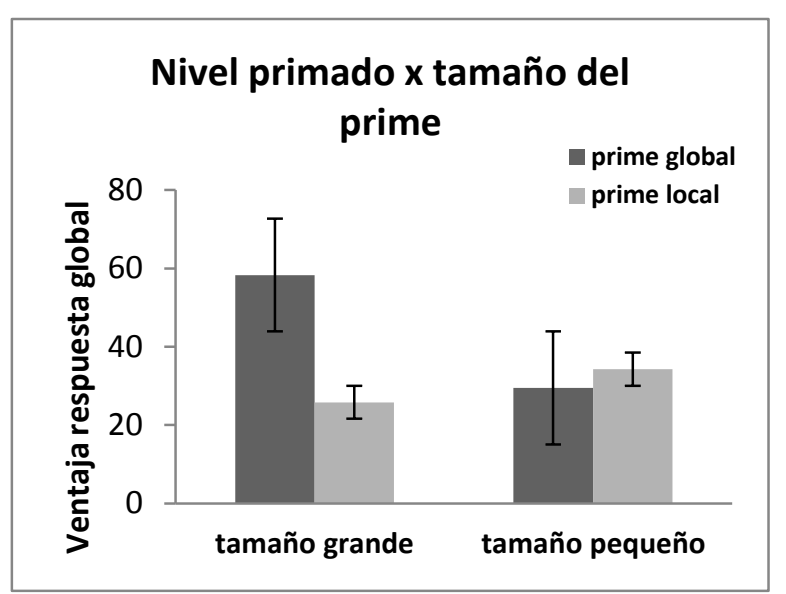

Figura 9. Experimento 2.Ventaja global en ms.

\section{Discusión}

El Experimento 2 extiende los resultados anteriores a un paradigma de atención dividida, con especial interés en las posibles diferencias debidas al cambio de tarea.

El primer dato a tener en cuenta es la existencia de un fuerte efecto de primacía global ausente en el Experimento 1. Una posible explicación para este cambio en la precedencia global podría encontrarse en los diferentes requisitos y distribución atencionales entre ambas tareas. Mientras el Experimento 1 posibilitaba que los participantes fijasen su atención en uno de los niveles jerárquicos del estímulo ignorando el nivel alternativo, eliminando de esta manera gran parte de la ventaja en las respuestas al nivel global, el Experimento 2 no permitía predecir de antemano el nivel jerárquico de aparición de los estímulos objetivo, lo que restauraría la ventaja en las respuestas al nivel global clásica en este tipo de tareas (Navon, 1977).

Más relevante aún es la interacción de segundo orden encontrada entre nivel primado, nivel de aparición del objetivo y tamaño absoluto del prime. Tras el cambio a una tarea de atención dividida el tamaño absoluto parece modular los efectos del nivel atencional primado. El efecto del nivel jerárquico atendido parece seguir el mismo patrón que en los experimentos anteriores, pero este efecto se restringe al tamaño mayor del prime en el que nivel atendido y tamaño absoluto coinciden. Las implicaciones de este resultado en relación al cambio de tarea, que contiene cierto paralelismo con los obtenidos por Kim et al. (1999), serán comentadas en profundidad en la Discusión general.

Los resultados tras el cálculo de la variable diferencial confirman los obtenidos con el análisis de los TRs. De nuevo la ventaja de las respuestas a los objetivos en el nivel global es mayor cuando se atiende previamente a este nivel respecto a cuándo se atiende de manera previa al nivel local, y de nuevo este efecto se encuentra circunscrito tan solo al tamaño absoluto mayor del prime.

En conclusión, los resultados del Experimento 2 replican el patrón de datos encontrados en el Experimento 1, aña- diendo un posible efecto modulador del tamaño absoluto del prime (o tamaño del foco atencional) cuando los participantes realizan una tarea de atención dividida. Sin embargo, el diseño de este experimento no permite clarificar cual es la verdadera naturaleza del efecto modulador del tamaño absoluto, que podría deberse al menos a tres posibles causas: (1) la falta de potencia en un paradigma de atención dividida del tamaño pequeño del prime para suscitar cualquier tipo de cambio en los TRs; (2) el efecto del tamaño absoluto del prime y no del nivel atendido; y (3) un efecto combinado del tamaño absoluto y del nivel atendido.

\section{Discusión general}

Los resultados del presente estudio apoyan la hipótesis de que el nivel jerárquico atendido (primado) es el responsable de la facilitación de las respuestas cuando el nivel primado coincide con el nivel en el que aparece el estímulo objetivo, y del aumento de la interferencia cuando ambos niveles no coinciden. Este resultado es especialmente visible en el Experimento 1 , en el que ningún efecto relacionado con el tamaño absoluto del prime fue relevante, y si lo fueron las interacciones relacionadas con el nivel atendido durante la tarea de prime. De especial interés es la replicación de este patrón de resultados cuando se tiene en cuenta tan solo el tamaño pequeño del prime en el que el tamaño o resolución total del estímulo coincide en tamaño con el nivel local del patrón jerárquico posterior. El hecho de que los efectos mencionados solo sean significativos para estímulos incongruentes sugiere que el mecanismo de actuación del priming atencional puede tener relación con la capacidad para reducir o aumentar la interferencia causada por el nivel complementario o alternativo al que aparece el objetivo.

Estos resultados, similares a los encontrados por Kim et al. (1999), ponen de manifiesto la existencia de moduladores atencionales del procesamiento visual de patrones jerárquicos. Los resultados del Experimento 2 incorporan ciertos matices relacionados con el cambio en los estímulos utilizados así como la tarea realizada (atención dividida). En particular, el efecto del nivel primado sobre el TR a los objetivos globales y locales se limita al tamaño mayor del prime. Resultados similares se encontraron en los experimentos 2 y 3 de Kim et al. (1999), en los que la adición de un marco o un nuevo nivel jerárquico que mantuviese constante el tamaño absoluto del estímulo, logró que el tamaño absoluto del estímulo atendido pasase a ser relevante para primar las respuestas de uno u otro nivel jerárquico. En nuestro estudio es el cambio de tarea desde un paradigma de atención selectiva a uno de atención dividida el que, a priori, parece responsable de la inclusión del tamaño absoluto como factor modulador de los resultados.

A pesar de que como se ha comentado en la discusión del Experimento 2, existen diferentes explicaciones para la restricción del efecto de priming secuencial al tamaño absoluto mayor del prime al pasar a una tarea de atención dividida, la variable diferencial calculada apunta a que esta restricción 
puede ser debida a un efecto del tamaño absoluto con independencia del nivel jerárquico atendido. Como puede observarse en la Figura 9, el grado de ventaja global cuando el tamaño absoluto del prime es pequeño permanece inalterado con independencia del nivel jerárquico sobre el que se dirija la atención y, aún más importante, este grado de ventaja global es similar al que se obtiene cuando se prima el nivel local en los estímulos de tamaño absoluto grande. Parece plausible por tanto, que tras el cambio de tarea sea el tamaño absoluto de la región del espacio atendida el responsable de los efectos de priming secuencial. Es difícil encontrar una explicación para este efecto obtenido con el cambio de paradigma, aunque se podría conjeturar de manera tentativa, que la ausencia de información previa sobre el nivel jerárquico del objetivo restaría importancia al nivel atendido con anterioridad y se la daría al tamaño absoluto de la región espacial atendida durante la tarea de priming, dando ventaja a los estímulos que coincidan en tamaño absoluto con los presentados durante esta tarea.

Un interesante recurso utilizado en el análisis de datos de ambos experimentos ha sido el cómputo de una variable diferencial, operativizada como ventaja (o desventaja) en milisegundos de las respuestas globales sobre las locales. Desde esta perspectiva, la ventaja global/local en el procesamiento de estímulos jerárquicos puede tomarse como un continuo desde la primacía local a la global y los estímulos utilizados pueden estar, en función de determinadas variables (intensidad del agrupamiento, tiempo de exposición, bondad de la forma, etc.), en un punto u otro de ese continuo hipotético. Con independencia de en qué punto del mismo se encuentren (incluso en los casos en que no existe ninguna clase de precedencia), el cálculo de una variable diferencial como la elaborada en el presente trabajo permite desentrañar la influencia del priming sobre las respuestas a objetivos en uno $\mathrm{u}$ otro nivel jerárquico, evitando que este efecto quede oculto para los análisis estadísticos tradicionales.

Los resultados obtenidos son consistentes con la existencia de mecanismos atencionales de alto nivel (huella atencional basada en el cómputo de frecuencias espaciales) que sesgan la atención hacia un extremo u otro del rango total de tamaños o resoluciones manteniendo al menos alguna información sobre sus proporciones relativas (Robertson 1996, 1999).

A pesar de que el presente experimento no manipula de manera explícita la frecuencia espacial de los estímulos, sus resultados pueden ser interpretados de manera tentativa en

\section{Referencias}

Arnau Gras, J., Blanca Mena, M., Rosel Remírez, J., y Salvador Beltrán, F. (1992). Direccionalidad del procesamiento de la información visual: una revisión desde la perspectiva cognitiva. Anales de Psicología, 8(1-2), 77-90. Doi:10.6018/analesps.8.1-2.28771

Blanca Mena, M., López Montiel, D., y López Montiel, G. (2006). Factores que Interactúan con la dominancia global en el procesamiento de estímulos jerárquicos. Anales De Psicología, 22(2), 273-289. Doi:10.6018/analesps.22.2.25981 términos de componentes de frecuencias espaciales (Flevaris y et al. 2011; Robertson, 1996; Shulman y Wilson, 1987). El hecho de que los elementos locales sean de menor tamaño que el conjunto del que forman parte implica que estos elementos se definen en el rango alto de frecuencias espaciales dentro de las relevantes para el análisis de los mismos, mientras que la información sobre los elementos globales está contenida en la banda de frecuencias espaciales más bajas. Una vez seleccionado el rango de frecuencias relevantes para el procesamiento del estímulo estas se procesan teniendo en cuenta la relación o proporción entre ellas, y esta información sobre las diferencias relativas en frecuencia espacial sería la responsable del efecto de priming encontrado. Ivry y Robertson (1998) propusieron un mecanismo de doble filtro de frecuencia (DFF) necesario para dar cuenta de la conversión de frecuencias espaciales absolutas en frecuencias espaciales relativas. El primer filtro seleccionaría el rango absoluto de frecuencias relevantes para la tarea mientras que el segundo filtraría las frecuencias relativas dentro de ese rango.

Este filtro mantendría su ajuste relativo tras la desaparición del estímulo utilizado como prime hasta la aparición del estímulo de prueba. Si el estímulo objetivo aparece entonces al mismo nivel, el ajuste previo del filtro concedería cierta ventaja a ese rango de frecuencias relativas cuando el mecanismo DFF actuase de nuevo (Kim et al. 1999; Robertson, 1996).

Como ya señalaron Kim et al. (1999), un mecanismo de este tipo proporcionaría una notable ventaja en el medio natural proporcionando una solución efectiva que permita mantener la atención sobre las características relevantes de un estímulo, con independencia de los cambios de tamaño de la imagen retiniana debidos al desplazamiento de los objetos o del observador por el ambiente. En un ambiente donde el organismo raramente encuentra estímulos proximales que permanezcan invariantes (no así los estímulos distales que permanecen relativamente estables), las ventajas adaptativas de un mecanismo de este tipo no deben obviarse.

Agradecimientos. Este trabajo ha sido posible gracias a la financiación con referencia 2012V/PUNED/0009 concedida por la UNED a Pedro R. Montoro y la ayuda con referencia FPU13/05914 concedida a Antonio Prieto por el Ministerio de Educación Cultura y Deporte. Los autores quieren expresar su agradecimiento a los profesores Marcos Ruiz, Antonio Crespo y Dolores Luna por sus valiosas opiniones, comentarios y correcciones durante la realización del manuscrito.

Bruce Henning, G., Gevene Hertz, B., y Broadbent, D. (1975). Some experiments bearing on the hypothesis that the visual system analyses spatial patterns in independent bands of spatial frequency. Vision Research, 15(8), 887-897.

Campbell, F. W., y Robson, J. (1968). Application of Fourier analysis to the visibility of gratings. The Journal of Physiology, 197(3), 551-566. 
Flevaris, A. V., Bentin, S., y Robertson, L. C. (2010). Local or global? Attentional selection of spatial frequencies binds shapes to hierarchical levels. Psychological Science, 21(3), 424-431.

Flevaris, A. V., Bentin, S., y Robertson, L. C. (2011). Attention to hierarchical level influences attentional selection of spatial scale. Journal of $E x-$ perimental Psychology: Human Perception and Performance, 37(1), 12-22. Doi: 10.1037/a0019251

Ivry, R. B., y Robertson, L. C. (1998). The two sides of perception. Cambridge, MA, US: The MIT Press.

Kim, N., Ivry, R. B., y Robertson, L. C. (1999). Sequential priming in hierarchically organized figures: Effects of target level and target resolution. Journal of Experimental Psychology: Human Perception and Performance; 25(3), 715-729. Doi: 10.1037/0096-1523.25.3.715.

Kimchi, R. (1992). Primacy of wholistic processing and global/local paradigm: A critical review. Psychological Bulletin, 112(1), 24-38. Doi: 10.1037/0033-2909.112.1.24

Kinchla, R., y Wolfe, J. (1979). The order of visual processing: "Topdown," "bottom-up," or "middle-out". Attention, Perception, \& Psychophysics, 25(3), 225-231

Lamb, M. R., y Yund, E. W. (1996). Spatial frequency and attention: Effects of level-, target-, and location-repetition on the processing of global and local forms. Attention, Perception, \& Psychophysics, 58(3), 363-373.

Lamb, M. R., y Yund, E. W. (1996). Spatial frequency and interference between global and local levels of structure. Visual Cognition, 3(3), 193-220. Doi: 10.1080/713756739

Lamb, M. R., Yund, E. W., y Pond, H. M. (1999). Is attentional selection to different levels of hierarchical structure based on spatial frequency? Journal of Experimental Psychology: General, 128(1), 88-94. Doi: 10.1037/0096-3445.128.1.88

Luna, D., Merino, J., y Marcos-Ruiz, R. (1990). Processing dominance of global and local information in visual patterns. Acta Psychologica, 73(2), 131-143.

Marí-Beffa, P., Fuentes, L. J., Catena, A., \& Houghton, G. (2000). Semantic priming in the prime task effect: Evidence of automatic semantic processing of distractors. Memory \& Cognition, 28(4), 635-647.

Mayas, J., Fuentes, L. J., \& Ballesteros, S. (2012). Stroop interference and negative priming (NP) suppression in normal aging. Archives of Gerontology and Geriatrics, 54(2), 333-338. Doi:10.1016/j.archger.2010.12.012
Montoro, P. R., \& Luna, D. (2009). Deconfounding the Effects of Local Element Spatial Heterogeneity and Sparsity on Processing Dominance. The Journal of General Psychology: Experimental, Psychological, and Comparative Psychology, 136(4), 407-427. Doi: 10.1080/00221300903269139

Navon, D. (1977). Forest before trees: The precedence of global features in visual perception. Cognitive Psychology, 9(3), 353-383.

Palmer, S. E. (1980). What makes triangles point: Local and global effects in configurations of ambiguous triangles. Cognitive Psychology, 12(3), 285305.

Palmer, S. E. (1999). Vision science: Photons to phenomenology. Cambridge, MA, US: The MIT Press.

Pomerantz, J. R. (1983). Global and local precedence: Selective attention in form and motion perception. Journal of Experimental Psychology: General, 112(4), 516-540. Doi: 10.1037/0096-3445.112.4.516.

Pomerantz, J. R., Sager, L. C., y Stoever, R. J. (1977). Perception of wholes and of their component parts: Some configural superiority effects. Journal of Experimental Psychology: Human Perception and Performance, 3(3), 422435. Doi: 10.1037/0096-1523.3.3.422

Robertson, L. C. (1996). Attentional persistence for features of hierarchical patterns. Journal of Experimental Psychology: General; 125(3), 227-249. Doi: 10.1037/0096-3445.125.3.227

Robertson, L. C. (1999). Spatial frequencies as a medium for guiding attention: Comment on Lamb, Yund, and Pond. Journal of Experimental Psychology: General, 128(1), 95-98. Doi: 10.1037/0096-3445.128.1.95

Robertson, L. C., Egly, R., Lamb, M. R., y Kerth, L. (1993). Spatial attention and cuing to global and local levels of hierarchical structure. Journal of Experimental Psychology: Human Perception and Performance, 19(3), 471-487. Doi: 10.1037/0096-1523.19.3.471

Robertson, L. C., Lamb, M. R., y Zaidel, E. (1993). Interhemispheric relations in processing hierarchical patterns: Evidence from normal and commissurotomized subjects. Neuropsychology, 7(3), 325-342. Doi: 10.1037/0894-4105.7.3.325

Shulman, G. L., y Wilson, J. (1987). Spatial frequency and selective attention to local and global information. Perception, 16(1), 89-101.

Ward, L. M. (1982). Determinants of attention to local and global features of visual forms. Journal of Experimental Psychology: Human Perception and Performance, 8(4), 562-581. Doi: 10.1037/0096-1523.8.4.562

(Articulo recibido: 09-05-2013; revisado: 11-01-2014; aceptado: 16-01-2014) 